\title{
Prospective evaluation of the utility of intraoperative confocal laser endomicroscopy in patients with brain neoplasms using fluorescein sodium: experience with 74 cases
}

\author{
Nikolay L. Martirosyan, MD, ${ }^{1}$ Jennifer M. Eschbacher, MD, ${ }^{2}$ M. Yashar S. Kalani, MD, PhD, ${ }^{1}$ \\ Jay D. Turner, MD, PhD, ${ }^{1}$ Evgenii Belykh, MD, ${ }^{1}$ Robert F. Spetzler, MD, ${ }^{1}$ Peter Nakaji, MD, and \\ Mark C. Preul, MD' \\ Departments of ${ }^{1}$ Neurosurgery and ${ }^{2}$ Neuropathology, Barrow Neurological Institute, St. Joseph's Hospital and Medical Center, \\ Phoenix, Arizona
}

OBJECTIVE This study evaluated the utility, specificity, and sensitivity of intraoperative confocal laser endomicroscopy $(C L E)$ to provide diagnostic information during resection of human brain tumors.

METHODS CLE imaging was used in the resection of intracranial neoplasms in 74 consecutive patients ( 31 male; mean age 47.5 years; sequential 10-month study period). Intraoperative in vivo and ex vivo CLE was performed after intravenous injection of fluorescein sodium (FNa). Tissue samples from CLE imaging-matched areas were acquired for comparison with routine histological analysis (frozen and permanent sections). CLE images were classified as diagnostic or nondiagnostic. The specificities and sensitivities of CLE and frozen sections for gliomas and meningiomas were calculated using permanent histological sections as the standard.

RESULTS CLE images were obtained for each patient. The mean duration of intraoperative CLE system use was 15.7 minutes (range 3-73 minutes). A total of 20,734 CLE images were correlated with 267 biopsy specimens (mean number of images/biopsy location, in vivo 84 , ex vivo 70 ). CLE images were diagnostic for $45.98 \%$ in vivo and $52.97 \%$ ex vivo specimens. After initiation of CLE, an average of 14 in vivo images and 7 ex vivo images were acquired before identification of a first diagnostic image. CLE specificity and sensitivity were, respectively, $94 \%$ and $91 \%$ for gliomas and $93 \%$ and $97 \%$ for meningiomas.

CONCLUSIONS CLE with FNa provided intraoperative histological information during brain tumor removal. Specificities and sensitivities of CLE for gliomas and meningiomas were comparable to those for frozen sections. These data suggest that CLE could allow the interactive identification of tumor areas, substantially improving intraoperative decisions during the resection of brain tumors.

http://thejns.org/doi/abs/10.3171/2016.1.FOCUS15559

KEY WORDS confocal laser endomicroscopy; fluorescein sodium; frozen section; intracranial neoplasms; tumor fluorescence

$\mathrm{T}$ HE frozen section biopsy is the standard method for intraoperative diagnosis in neurosurgery. Histological interpretation is based on relatively small volumes of tissues from a usually limited number of biopsy specimens. The complete process of transfer of tissue and waiting time for diagnostic evaluation often requires up to 40 minutes. Moreover, compared with permanent sec- tions, frozen sections often do not reveal the histological features necessary for the final diagnosis; this is due to many factors, including frozen section artifact and sampling error. ${ }^{1,11}$ The diagnostic discrepancy between frozen and permanent sections is reported to be as high as $2.7 \%$ for intracranial pathology. ${ }^{12}$ Thus, permanent sections are the current diagnostic standard, yet they require several

ABBREVIATIONS CLE = confocal laser endomicroscopy; $F N=$ false negative; $F N a=$ fluorescein sodium; $F P=$ false positive; $H$ \& $E=$ hematoxylin and eosin; $T N=$ true negative; TP = true positive; WHO = World Health Organization 
days to process for interpretation. Such conventional sampling of tissue and interpretation of histology is impractical for interactive guidance of resection.

Fluorescence imaging techniques offer an alternative means for acquiring intraoperative data and have the advantage of rapid acquisition for enhancing real-time decision making. ${ }^{6,7}$ Intraoperative confocal laser endomicroscopy (CLE) has the potential to facilitate surgical work flow through rapid digital image screening of multiple observational fields. ${ }^{2,19}$ This benefit was validated in its application to gastrointestinal and gynecological surgery. ${ }^{5,15-18}$ We have investigated the feasibility of CLE in a murine malignant glioma model to distinguish normal brain, microvasculature, and tumor margins.$^{4,8,9,14}$ Furthermore, we have demonstrated its potential application as an intraoperative tool in human brain tumor surgery., ${ }^{3,13}$ In the current study, we sought to evaluate the utility, specificity, and sensitivity of CLE using fluorescein sodium (FNa) as a fluorophore to detect brain tumor tissue. To the best of our knowledge, this is the first attempt at in-depth diagnostic analysis of CLE for a brain tumor application.

\section{Methods}

This prospective study evaluated our clinical experience using CLE during resection of intracranial masses over a sequential 10-month period at the Barrow Neurological Institute, St. Joseph's Hospital and Medical Center, Phoenix, Arizona. Patients with a preoperative diagnosis of a brain tumor requiring surgical intervention were prospectively enrolled in this study, which was approved by the institutional review board. The study period was defined by CLE system availability. Patient exclusion criteria were as follows: inability to give signed informed consent, age younger than 18 years, previous documented adverse reaction or allergy to $\mathrm{FNa}$, pregnancy, and current usage of angiotensin-converting enzyme inhibitors or beta-blockers.

\section{Imaging Protocol}

The CLE (Optiscan 5.1, Optiscan Pty, Ltd.) image acquisition parameters have been described in detail in our previous reports. ${ }^{3,8,9}$ Briefly, this system consists of a handheld miniaturized optical laser scanner designed as a rigid probe with a $6.3-\mathrm{mm}$ outer diameter and a working length of $150 \mathrm{~mm}$. A 488-nm diode laser provided incident excitation light, and fluorescent emission was detected at 505$585 \mathrm{~nm}$ using a band-pass filter, via a single optical fiber acting as both the excitation pinhole and the detection pinhole for confocal isolation of the focal plane. The detector signal was digitized synchronously with the scanning to construct images parallel to the tissue surface (en face optical sections). Laser power was typically set to 550$900 \mu \mathrm{W}$ at brain tissue; maximum power was limited to $1000 \mu \mathrm{W}$. A field of view of $475 \times 475 \mu \mathrm{m}$ (approximately 1000x magnification on a 21 -inch screen) was scanned either at $1024 \times 512$ pixels $(0.8 /$ second frame rate $)$ or at $1024 \times 1024$ pixels (1.2/second frame rate), with a lateral resolution of $0.7 \mu \mathrm{m}$ and an axial resolution (i.e., effective optical slice thickness) of approximately $4.5 \mu \mathrm{m}$. The resulting images were stored digitally and could be recorded as a time-lapse series producing essentially a digital film loop. During the procedure, a foot pedal provided remote control of the variable confocal imaging plane depth from the surface to a depth of $0-500 \mu \mathrm{m}$.

In vivo images were acquired 5 minutes after intravenous injection of $5 \mathrm{ml} \mathrm{10 \%} \mathrm{FNa.} \mathrm{The} \mathrm{majority} \mathrm{of} \mathrm{images}$ were obtained by using the CLE probe affixed to a Greenberg retractor arm. The retractor was tightened to a degree that allowed both smooth movement and steady operation. The probe was moved gently, without losing contact, along the surface of the tissue to obtain images from several biopsy locations. One neurosurgeon used the probe mainly in a freehand fashion-not affixed to a holder or retractor arm. Co-registration of the probe with the image guided surgical system permitted precise intraoperative localization of the CLE imaging with the site of the biopsy.

Locations of imaging included normal brain and regions of obvious tumor, in addition to the transitional zone between what appeared to be normal brain and tumor. Images were acquired from each biopsy location. Intraoperative CLE was performed by 4 neurosurgeons.

\section{Tissue Sampling, Histology, and Data Processing}

For in vivo imaging, multiple locations within the resection bed were imaged with CLE. Tissue samples (approximately $0.5 \mathrm{~cm}^{3}$ ) were harvested from each patient during the procedure. For ex vivo imaging purposes, tissue samples suspicious for tumor were harvested from the surgical field and imaged on a separate work station away from the patient, but within the operating room. No additional fluorophore beyond intravenous FNa was used for ex vivo imaging. Tissue samples were placed on gauze and imaged using the probe in a freehand fashion. Multiple images were obtained from each biopsy location.

Areas that were imaged using CLE were marked with tissue ink so that precise locations could be validated with conventional histology. The tissue was placed in a cassette for standard formalin fixation and paraffin embedding. Histological assessment was performed using standard light microscopic evaluation of $10-\mu \mathrm{m}$-thick hematoxylin and eosin ( $\mathrm{H} \& \mathrm{E})-$ stained sections. CLE images were compared with both frozen and permanent histological sections.

Images were reviewed by a neuropathologist and 2 neurosurgeons who were not involved in the surgeries. For each case, they analyzed the histopathological features of corresponding CLE images and $\mathrm{H}$ \& E-stained frozen and permanent sections. Images were classified as diagnostic (i.e., the confocal images revealed identifiable histological features) or as nondiagnostic (i.e., the image provided no identifiable histological features due to distortion by blood artifact [erythrocytes] or motion artifact) (Fig. 1). The numbers of diagnostic and nondiagnostic images were quantified and analyzed. Other variables that were studied included the duration of operation of the CLE, the acquisition of images, and how each individual surgeon operated the system.

\section{Sensitivity and Specificity}

The interpretation of CLE images and frozen sections 

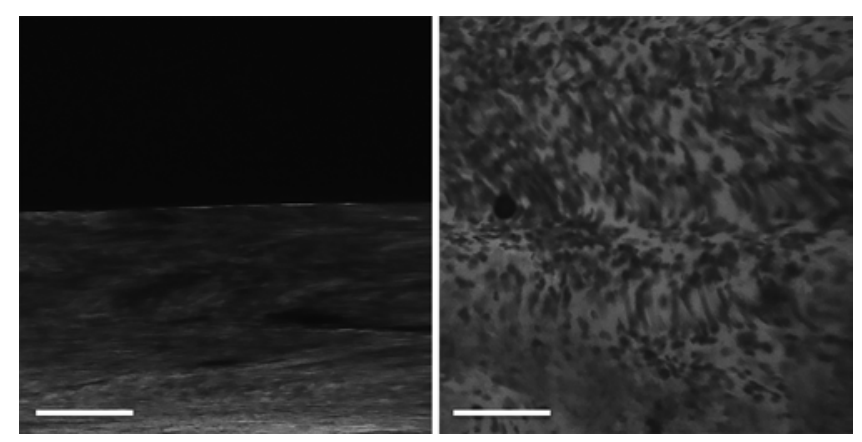

FIG. 1. In vivo CLE images demonstrating motion artifact (left) and red blood cells (right). Bar $=100 \mu \mathrm{m}$.

was compared with information from permanent histological sections. True-negative (TN) and false-negative (FN) values, as well as true-positive (TP) and false-positive (FP) values, were calculated. Sensitivity was defined as $\mathrm{TP} /(\mathrm{TP}+\mathrm{FN})$, and specificity was defined as $\mathrm{TN} /(\mathrm{TN}+\mathrm{FP})$. The specificity and sensitivity were determined for in vivo CLE images and frozen $\mathrm{H} \& \mathrm{E}$-stained sections for diagnosing meningiomas and gliomas, which were the 2 most common types of pathology in the series and allowed satisfactory statistical analyses. The diagnostic interpretation of permanent histological sections served as the "gold standard."

\section{Statistical Analysis}

Statistical analysis was performed with SPSS Statistics for Windows, version 18 (SPSS, Inc.). Pearson's correlation coefficient was calculated to assess the correlation between the number of diagnostic images and the duration of imaging time. Analysis of variance was used to analyze variation of diagnostic images by tumor type. The mean number of images taken before the first diagnostic image was determined for each patient. A $p$ value $\leq 0.05$ was considered significant. Means are presented \pm SD.

\section{Results}

\section{Image Analysis}

Seventy-four consecutive adult patients (31 male and 43 female) were prospectively enrolled in the study. Their mean age at presentation was 47.5 years (range 18-81 years).

A total of 20,734 confocal images were acquired, including 11,920 in vivo and 8814 ex vivo. A total of 267 biopsy specimens were obtained, including 141 that were imaged in vivo and 126 imaged ex vivo. There were only 2 cases (one involving a patient with a craniopharyngioma and the other a patient with a chordoma) in which in vivo images were not obtained (Tables 1, 2, and 3). Ex vivo imaging was performed in every case.

The mean imaging time, defined as the total time of use of the device in the operating room, was 5.8 minutes per patient (range 1.4-17.0 minutes). The mean number of images taken per patient was 280 . Each patient had a mean of 3 (range 2-9) in vivo biopsy locations. These areas were imaged in vivo with CLE, and corresponding tissue samples were processed for histological evaluation. The mean number of images taken from each in vivo biopsy location
TABLE 1. Number of confocal endomicroscopy images*

\begin{tabular}{lccc}
\hline \multicolumn{1}{c}{ Variable } & Total & Ex Vivo & In Vivo \\
\hline Total no. of images & 20,734 & 8814 & 11,920 \\
\hline $\begin{array}{c}\text { Mean no. of images } \\
\text { per pt }\end{array}$ & $280.2 \pm 175.2$ & $119.1 \pm 86.8$ & $161.1 \pm 125.7$ \\
\hline $\begin{array}{c}\text { Total no. of biopsy } \\
\text { specimens }\end{array}$ & 267 & 126 & 141 \\
\hline $\begin{array}{c}\text { Mean no. of biopsy } \\
\text { specimens per pt }\end{array}$ & $3.6 \pm 1.7$ & $1.7 \pm 0.8$ & $1.9 \pm 1.3$ \\
\hline $\begin{array}{c}\text { Mean no. of images } \\
\text { per biopsy specimen }\end{array}$ & $77.7 \pm 46.2$ & $70.0 \pm 39.6$ & $84.5 \pm 50.6$ \\
\hline
\end{tabular}

$\mathrm{Pt}=$ patient.

${ }^{*}$ Mean values are presented \pm SD.

was 84 (range 7-252). The majority of patients had 1 or 2 biopsy specimens imaged using ex vivo methodology (Table 3). The mean number of images acquired from each ex vivo biopsy specimen was 70 (range 20-244) (Table 1).

Totals of 5481 (46.0\%) in vivo and 4590 (52.1\%) ex vivo diagnostic confocal images were acquired (Table 2). In cases of glioma, the mean number of images taken per patient was 372 , which was significantly higher than for patients with meningiomas (mean 206 images) or schwannomas (mean 175 images) (Fig. 2A). The percentage of diagnostic in vivo or ex vivo images acquired did not vary by diagnosis.

After initiation of CLE, on average, 14 images for in vivo and 7 images for ex vivo CLE were acquired before a first diagnostic image was identified (Fig. 2B). There was no statistically significant difference in the total number of images taken for each biopsy site for gliomas and meningiomas (Fig. 2C). Analysis showed a positive correlation between the number of diagnostic images and imaging time (Pearson's correlation coefficient $\mathrm{R}^{2}=0.61$, $\mathrm{p}<$ 0.001) (Fig. 2D).

\section{Specificity and Sensitivity}

The CLE specificity and sensitivity for gliomas were $94 \%$ and $91 \%$, respectively. The frozen section specificity and sensitivity for gliomas were $96 \%$ and $95 \%$, respectively. Both FN and FP values were evident in situations of attempting to distinguish reactive changes and recurrent glioma. FN and FP values for meningiomas are associated only with distinguishing between meningioma and schwannoma (Table 4).

TABLE 2. Distribution of diagnostic and nondiagnostic images*

\begin{tabular}{|c|c|c|c|c|}
\hline Image Type & Max & $\begin{array}{c}\text { Sum } \\
\text { (for all pts) }\end{array}$ & $\%$ & Mean per Pt \\
\hline Diagnostic ex vivo images & 333 & 4590 & 52.07 & $62.03 \pm 2.07$ \\
\hline $\begin{array}{l}\text { Nondiagnostic ex vivo } \\
\text { images }\end{array}$ & 233 & 4224 & 47.92 & $57.08 \pm 50.99$ \\
\hline Diagnostic in vivo images & 380 & 5481 & 45.98 & $76.12 \pm 68.04$ \\
\hline $\begin{array}{l}\text { Nondiagnostic in vivo } \\
\text { images }\end{array}$ & 334 & 6439 & 54.01 & $89.43 \pm 80.54$ \\
\hline
\end{tabular}

\footnotetext{
${ }^{*}$ Mean values are presented \pm SD.
} 
TABLE 3. Number of in vivo and ex vivo biopsies

\begin{tabular}{cc}
\hline In Vivo, No. (\%) & Ex Vivo, No. (\%) \\
\hline $0(2.7)$ & - \\
\hline $1(45.9)$ & $1(48.6)$ \\
\hline $2(28.4)$ & $2(36.5)$ \\
\hline $3(13.5)$ & $3(10.8)$ \\
\hline $4(4.1)$ & $4(4.1)$ \\
\hline $5(2.7)$ & - \\
\hline $6(1.4)$ & - \\
\hline $7(1.4)$ & - \\
\hline
\end{tabular}

* Percentages represent proportions of cases and total more than $100 \%$ due to rounding.

\section{Histopathological Analysis}

Seven of the 74 patients who were imaged had no intracranial tumor (Fig. 2E). Tumor tissue was not detected with either CLE or with conventional histological methods, including in 1 case of glioblastoma-associated postradiation treatment effect. One patient had cortical dysplasia, 6 had reactive gliosis, and 1 patient had sarcoidosis. Sixty-six patients had brain neoplasms requiring surgical treatment. Of the 66 tumors, 30 were meningiomas (World Health Organization [WHO] Grade I [ $=25]$, II [n = 4], and III [n $=1]$ ). Gliomas were diagnosed in 21 cases (WHO Grade II $[\mathrm{n}=8]$, Grade III [ $\mathrm{n}=10]$, and Grade IV [ $\mathrm{n}=3]$ ). The remaining 15 cases were distributed as follows: 4 schwannomas, 2 craniopharyngiomas, 2 hemangioblastomas, and 1 each for metastasis, subependymal giant cell astrocytoma, ependymoma, myxopapillary ependymoma, gliosarcoma, chordoma, and hemangioma. Pathological subtype information originated from histopathological evaluation.

\section{Confocal Laser Endomicroscopy}

Unique aspects of individual cells and other surrounding tumor tissue were observed as the CLE probe acquired images throughout its focal-depth range. In general, CLE of the FNa distribution revealed striking microvascular and extravascular structures, including cellular and subcellular structures in various tumor regions that correlated with the matched histological sections and known tissue architecture, such as pleomorphism, atypia, and hypercellularity contrasting against the fluorescent extracellular background. The FNa fluorescence intensity dynamics across tumor cells showed delineation of nuclei appearing darker than cytoplasm. Such a pattern of cell structure appearance could be defined in most cases. Importantly, individual tumor cells within brain tissue could be identified and a tumor border could often be delineated. The FNa image demonstrates cells in silhouetted appearance with intracellular components appearing as shadows of varying grayscale intensities. In 3 glioma cases, prominent tumoral satellitosis was seen. Extracellular matrix and tissue architectural features were difficult to conclusively identify. Erythrocytes were visualized as multiple, regularly shaped cells that appeared to be smaller than tumor cells.

Imaging of meningiomas demonstrated patterns characteristic of their subtype, including whorls and nests of cells likely corresponding to epithelial cells and bands of spindle cells. Tissue structures consistent with collagen and psammoma bodies were frequently identified. Grade II and Grade III meningiomas appeared to demonstrate more atypical cellular morphology than Grade I tumors, although this differentiation was difficult to identify consistently (Fig. 3).

Overall, typical histological characteristics of highgrade gliomas as noted on routine histological examination were identified on matched CLE images. Within obvious tumor tissue, images were dominated by extravascular FNa concentrated in clusters and nests around cellular structures showing hypercellularity and pleomorphism, which are morphologically consistent with extracellular fluorescence around and highlighting tumor cells (Fig. 4).

Imaging of craniopharyngiomas revealed structures consistent with wet keratin admixed with nests of cells with epithelial features. Imaging of hemangioblastomas revealed thin-walled blood vessels with interposed xanthomatous stromal cells. Multinucleated giant cells were identified in the case of sarcoidosis. Schwannomas exhibited a fasicular architecture composed of spindled cells with elongated cytoplasmic processes. Unique images of cell processes were obtained in a subependymal giant cell astrocytoma (Figs. 5 and 6).

The images acquired with the CLE probe affixed to the retractor system were initially higher in quality, having less artifact, than images acquired by handheld operation of the probe. However, as neurosurgeons gained experience with operation of the probe, they obtained higher-quality images. Handheld use of the CLE system was more facile. As the study progressed, the CLE system was used more to detect tumor boundaries than to acquire images of obvious tumor tissue from the core of the mass.

\section{Discussion}

This prospective clinical study describes the utility of CLE using FNa for diagnostic assessment of intracranial tumors. To our knowledge, this is the first analysis of the specificity and sensitivity of CLE in a human brain tumor application. Intraoperative CLE produced a large number of diagnostic digital images in a relatively short time. In vivo imaging provided real-time diagnostic intraoperative histopathological information in most cases. Ex vivo imaging of biopsy specimens was also an effective and relatively fast method for gathering diagnostic digital images without interfering with ongoing surgery.

In this study, the mean total imaging time was 5.8 minutes per case. This duration was sufficient to image a mean of 4 biopsy locations per patient (Table 3). In comparison, 1 frozen-section biopsy often requires up to 20 minutes or longer for complete processing and diagnostic interpretation. ${ }^{1}$ Further optimization of CLE image acquisition parameters and operative work flow can potentially reduce procedure time. ${ }^{10}$

\section{CLE Diagnostic Utility}

The specificity and sensitivity of CLE for the diagnosis of gliomas and meningiomas produced results comparable to those of frozen sections (Table 4). With further improvement of the CLE technology and implementation 

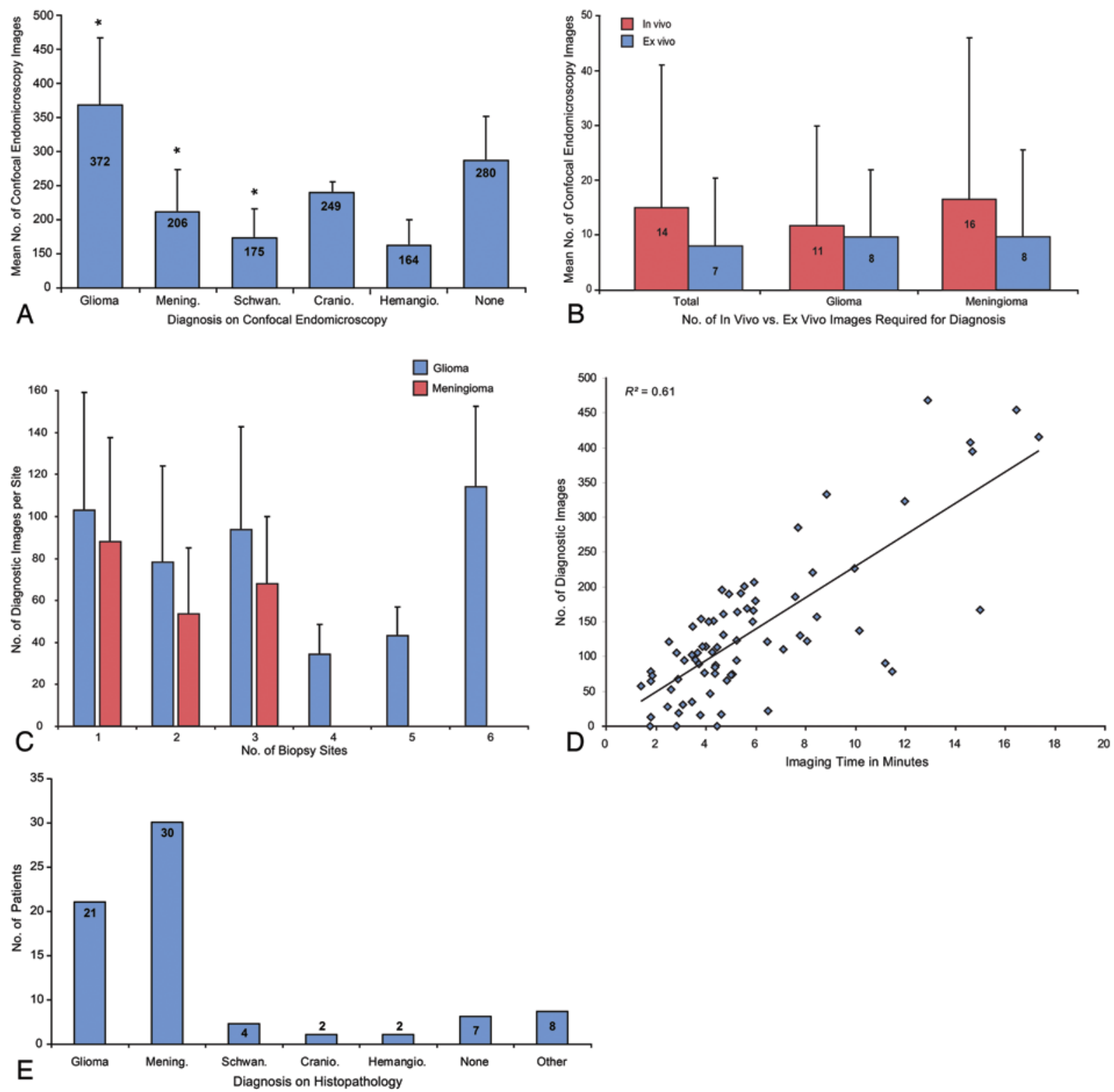

FIG. 2. A: Mean number of CLE images by type of diagnosis. Asterisks indicate that the mean total number of images taken for gliomas was more than the number taken for either meningiomas or schwannomas $(p=0.01)$. Bars indicate standard deviations. B: Mean number of CLE images acquired until first diagnostic image was determined for all diagnoses and for gliomas and meningiomas. Bars indicate standard deviations. C: Number of diagnostic images per biopsy site for gliomas and meningiomas. Gliomas required more imaging sites due to the complexity of image interpretation. D: Scatterplot showing correlation between the number of informative confocal endomicroscopy diagnostic images and the duration of time required to obtain them $\left(R^{2}=0.61\right.$, $p$ < 0.001). E: Number of histopathological diagnoses in the 74 patients group. Cranio. $=$ craniopharyngioma; Hemangio. $=$ hemangioblastoma; Mening. = meningioma; Schwan. = schwannoma.

of additional fluorophores that can be used in vivo and ex vivo (such as those that more specifically stain intracellular structures), sensitivity and specificity would be expected to increase. A lower number of false results with meningiomas were associated with better discrimination of specific histopathological features (e.g., whorls, collagen, psammoma bodies). Sensitivity and specificity for other tumor types was not assessed due to the relatively small number of these pathologies in our series.

Despite a relatively large number of nondiagnostic in vivo images (6439), the classification of diagnostic and nondiagnostic image groups is rather artificial, because all images were collected as a whole set. The impression for diagnosis was made by viewing the set, although usually 1 diagnostic image per biopsy specimen easily contributed to interpretations and decisions about tissue architecture and the differentiation of tumor from normal brain tissue (Table 2). Although many uninformative images may be acquired, informative images acquired even after many uninformative images still yield usable diagnostic or tissue characteristic information more rapidly than conventional histopathological frozen section interpretation. 
TABLE 4. Specificity and sensitivity of frozen sections and confocal endomicroscopy to detect gliomas and meningiomas with reference to permanent histological sections

\begin{tabular}{ccc}
\hline & \multicolumn{2}{c}{ Tumor Type } \\
\cline { 2 - 3 } Evaluation & Glioma & Meningioma \\
\hline Frozen section & & $98 \%$ \\
\hline Specificity & $96 \%$ & $97 \%$ \\
\hline Sensitivity & $95 \%$ & \\
\hline CLE & & $93 \%$ \\
\hline Specificity & $94 \%$ & $97 \%$ \\
\hline Sensitivity & $91 \%$ &
\end{tabular}

Approximately the 14th image acquired in vivo and the 7th image acquired ex vivo were diagnostic. This finding shows consistent results in acquiring diagnostic images from the initiation of imaging within 17 seconds for in vivo use and within 8 seconds for ex vivo use (Fig. 2A). More time was required to obtain the first diagnostic in vivo image than the first diagnostic ex vivo image. This difference was likely due to motion and blood artifacts decreasing diagnostic yield for CLE in vivo. The average time required to obtain the first diagnostic image after initiation of CLE did not vary by diagnosis for either in vivo or ex vivo imaging. This finding demonstrates that CLE was more or less equally diagnostic for all imaged cases. There was a positive correlation between the number of diagnostic images and imaging time, indicating that longer duration of imaging yields a greater number of diagnostic images.

There is a learning curve in using this CLE system. The probe position should be changed multiple times to acquire optimal images from a given area. It was not fea-
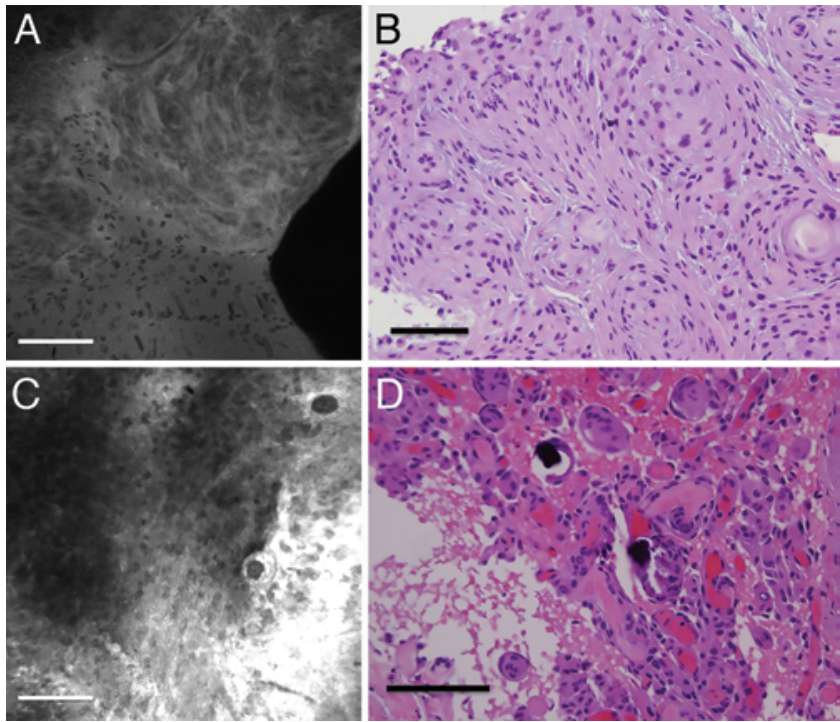

FIG. 3. In vivo CLE images ( $A$ and $\mathbf{C}$ ) obtained in 2 cases demonstrating transitional meningioma after $\mathrm{FNa}$ injection and photomicrographs of $H$ \& $E$-stained sections ( $B$ and $D$ ) showing histopathological features of whorls, psammoma bodies, and collagen similar to those of the matched imaging location. Bar $=100 \mu \mathrm{m}$. Original magnification $\times 200(\mathrm{~B})$ and ×400 (D).
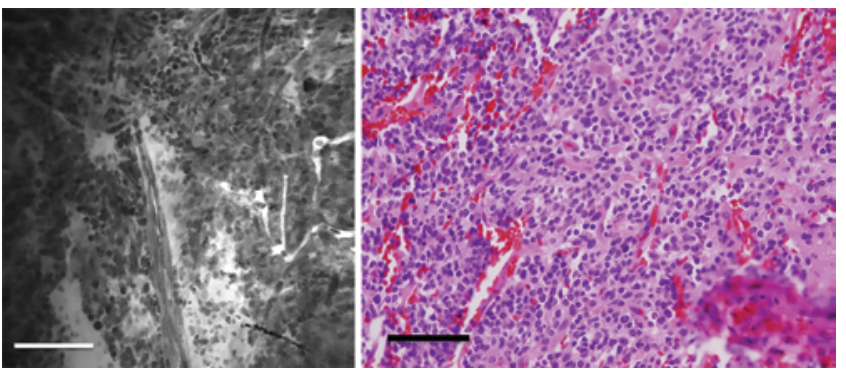

FIG. 4. Left: In vivo CLE image demonstrating an anaplastic oligodendroglioma after FNa injection. Right: Photomicrograph of tissue from the same imaging location showing cellular tumor with similar histopathological features of hypercellularity, atypia, and neovascularization. $\mathrm{H} \& \mathrm{E} . \mathrm{Bar}=100 \mu \mathrm{m}$. Original magnification $\times 200$.

sible to image the entire resection cavity due to the small field of view of the CLE $(475 \times 475 \mu \mathrm{m})$. However, there is no practical necessity to screen the entire surgical bed. The design of the technology is to acquire "optical biopsies" in selected regions that yield high-value information. This CLE system appears helpful for imaging of selected tumor regions, portions of the resection cavity, suspicious regions, and tumor boundaries. CLE is helpful to optically identify the extent of brain region involvement of an infiltrating mass. CLE was capable of producing images that clearly and rapidly showed tissue with tumor versus nontumor characteristics. The interpretation of the black, gray, and white digital FNa images compared with inspection of standard $\mathrm{H} \& \mathrm{E}-$ stained tissue preparations requires experience with CLE. The CLE system used in this study acquires images at the rate of 1.2 second per frame. Multiple image frames can be acquired from a single biopsy sample $\left(0.5 \mathrm{~cm}^{3}\right)$. Minor movements of the CLE probe within the same tissue region produced different images; thus there are multiple matched images from a single biopsy specimen location. Unlike in vivo application, ex vivo imaging could be performed by a technician within the operating room, without interrupting the flow of the surgical procedure.

Motion and blood artifacts, especially with FNa CLE, may decrease diagnostic yield. However, the probe lens
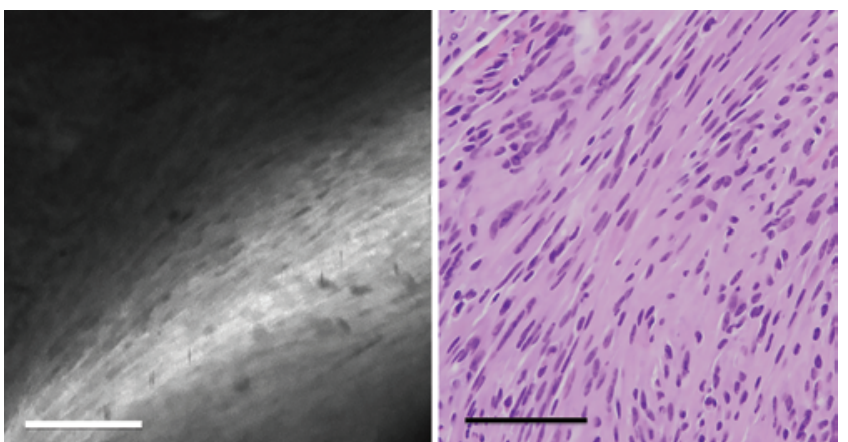

FIG. 5. Left: In vivo CLE image demonstrating a schwannoma after FNa injection. Right: Photomicrograph of tissue from the same imaging location showing cellular tumor with similar histopathological features of fascicles of cells with elongated cytoplasmic processes of Antoni A regions. Vague nuclear detail could be appreciated and consisted of dark oval intracytoplasmic structures that conformed with cell shape. $H$ \& E. Bar $=100 \mu \mathrm{m}$. Original magnification $\times 400$. 


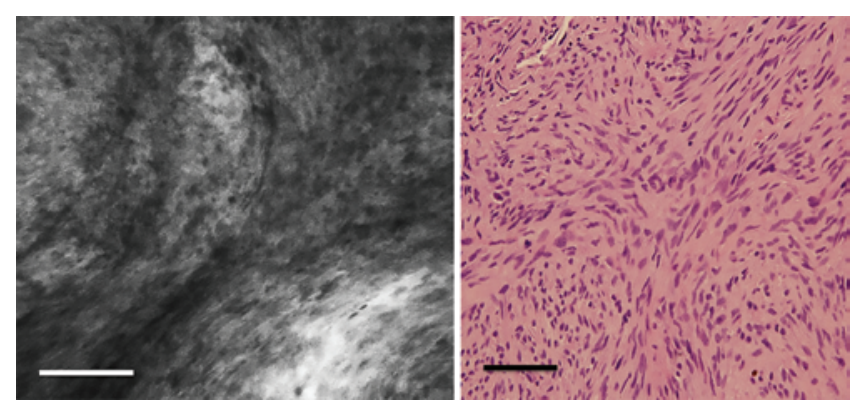

FIG. 6. Left: Ex vivo CLE image demonstrating a gliosarcoma after $\mathrm{FNa}$ injection. Right: Photomicrograph of tissue from the same imaging region showing cellular tumor with histopathological features similar to those of the gliosarcoma, demonstrating fascicles of markedly atypical elongated tumor cells, which appeared dark against a bright fluorescent background. Prominent collagen structure was also appreciated. H \& E. Bar $=100 \mu \mathrm{m}$. Original magnification $\times 200$.

can be easily and rapidly cleaned by irrigation or wiping. Motion artifacts could be overcome with the design of an improved probe holder. Moreover, with practice, the surgeons in our study could easily move and manipulate the probe to acquire diagnostic images, which is a significant advantage provided by such imaging technology. Compared with in vivo imaging, ex vivo imaging showed less blood or motion artifact, providing clearer image quality.

\section{Limitations of CLE With FNa}

An exact histopathological diagnosis was not identified for all cases using CLE. Such was possible only for masses with distinct histopathological features (e.g., psammoma bodies, collagen, spindle cells, multinucleated giant cells, wet keratin, and corpora amylacea). Although CLE showed distinct regions of hypercellularity, atypia, and tumor satellitosis, it was not possible to distinguish gliomas and reactive tissue changes in all cases. This situation is related largely to the use of FNa, which did not provide enough contrast or clarity to reliably distinguish and accurately count mitoses. Most glioma cases were difficult to classify according to WHO grade. A significantly higher number of confocal images was acquired for gliomas for both in vivo and ex vivo imaging. Surgeons acquired more images in an attempt to acquire diagnostic information.

The rigid design of the current CLE system probe may not provide optimal functionality in deep or remote regions of the operating field, especially in cases in which retractorless or handheld techniques are used. In our case series, 2 patients had lesions located in deep eloquent brain regions. These tumors were resected through narrow surgical corridors, and it was challenging in those cases to satisfactorily navigate the CLE probe. In the current configuration of the CLE system, in vivo human brain use is limited by the number of fluorophores detectable in the system's laser spectrum (505-585 nm). Only FNa is approved for in vivo use with this CLE system, whereas in the gastrointestinal tract, various fluorophores (e.g., cresyl violet or acriflavine) can be used. However, these fluorophores can be used in ex vivo mode on brain tissue to provide images with more specific cellular and subcellular information. Further development of CLE systems should also be directed toward improvement of image resolution, multiple or adjustable excitation/detection spectra, faster image acquisition time, larger field of view, and increased power and stability of the laser system.

\section{Conclusions}

Intraoperative CLE using FNa provided real-time in vivo and ex vivo histological information precisely related to the site of tissue imaging and for tissue biopsy. Although FNa may not provide specific tumor labeling, the specificity and sensitivity of CLE to diagnose gliomas and meningiomas were comparable to those of frozen section, using permanent histological sections as the standard. These data suggest that in vivo and ex vivo CLE could allow the interactive identification of tumor areas, substantially improving intraoperative decisions during the resection of brain tumors and radically changing the neurosurgeryneuropathology work flow in the operating room.

Investigations to discover clinically safe and more specific fluorescent stains, labels, or probes for brain applications are required. Further development of CLE systems should address improvements in image resolution and acquisition parameters.

\section{Acknowledgments}

We thank Guido Hattendorf of Carl Zeiss Surgical GmbH for expert technical assistance. We thank the Neuroscience Publications staff of Barrow Neurological Institute for assistance in preparing this manuscript.

\section{References}

1. Ackerman LV, Ramirez GA: The indications for and limitations of frozen section diagnosis; a review of 1269 consecutive frozen section diagnoses. Br J Surg 46:336-350, 1959

2. Behbahaninia M, Martirosyan NL, Georges J, Udovich JA, Kalani MY, Feuerstein BG, et al: Intraoperative fluorescent imaging of intracranial tumors: a review. Clin Neurol Neurosurg 115:517-528, 2013

3. Eschbacher J, Martirosyan NL, Nakaji P, Sanai N, Preul MC, Smith KA, et al: In vivo intraoperative confocal microscopy for real-time histopathological imaging of brain tumors. J Neurosurg 116:854-860, 2012

4. Fenton KE, Martirosyan NL, Abdelwahab MG, Coons SW, Preul MC, Scheck AC: In vivo visualization of GL261-luc2 mouse glioma cells by use of Alexa Fluor-labeled TRP-2 antibodies. Neurosurg Focus 36(2):E12, 2014

5. Fuchs FS, Zirlik S, Hildner K, Frieser M, Ganslmayer M, Schwarz S, et al: Fluorescein-aided confocal laser endomicroscopy of the lung. Respiration 81:32-38, 2011

6. Georges J, Zehri A, Carlson E, Nichols J, Mooney MA, Martirosyan NL, et al: Label-free microscopic assessment of glioblastoma biopsy specimens prior to biobanking. Neurosurg Focus 36(2):E8, 2014 [Erratum in Neurosurg Focus 36(6): Erratum, 2014]

7. Georges JF, Martirosyan NL, Eschbacher J, Nichols J, Tissot M, Preul MC, et al: Sulforhodamine 101 selectively labels human astrocytoma cells in an animal model of glioblastoma. J Clin Neurosci 21:846-851, 2014

8. Martirosyan NL, Cavalcanti DD, Eschbacher JM, Delaney PM, Scheck AC, Abdelwahab MG, et al: Use of in vivo nearinfrared laser confocal endomicroscopy with indocyanine green to detect the boundary of infiltrative tumor. J Neurosurg 115:1131-1138, 2011

9. Martirosyan NL, Georges J, Eschbacher JM, Cavalcanti DD, Elhadi AM, Abdelwahab MG, et al: Potential application of 
a handheld confocal endomicroscope imaging system using a variety of fluorophores in experimental gliomas and normal brain. Neurosurg Focus 36(2):E16, 2014

10. Neumann H, Vieth M, Atreya R, Neurath MF, Mudter J: Prospective evaluation of the learning curve of confocal laser endomicroscopy in patients with IBD. Histol Histopathol 26:867-872, 2011

11. Novis DA, Zarbo RJ: Interinstitutional comparison of frozen section turnaround time. A College of American Pathologists Q-Probes study of 32868 frozen sections in 700 hospitals. Arch Pathol Lab Med 121:559-567, 1997

12. Plesec TP, Prayson RA: Frozen section discrepancy in the evaluation of central nervous system tumors. Arch Pathol Lab Med 131:1532-1540, 2007

13. Sanai N, Eschbacher J, Hattendorf G, Coons SW, Preul MC, Smith KA, et al: Intraoperative confocal microscopy for brain tumors: a feasibility analysis in humans. Neurosurgery 68 (2 Suppl Operative):282-290, 2011

14. Sankar T, Delaney PM, Ryan RW, Eschbacher J, Abdelwahab M, Nakaji P, et al: Miniaturized handheld confocal microscopy for neurosurgery: results in an experimental glioblastoma model. Neurosurgery 66:410-418, 2010

15. Sonn GA, Jones SN, Tarin TV, Du CB, Mach KE, Jensen KC, et al: Optical biopsy of human bladder neoplasia with in vivo confocal laser endomicroscopy. J Urol 182:1299-1305, 2009

16. Tan J, Quinn MA, Pyman JM, Delaney PM, McLaren WJ: Detection of cervical intraepithelial neoplasia in vivo using confocal endomicroscopy. BJOG 116:1663-1670, 2009

17. van den Broek FJ, van Es JA, van Eeden S, Stokkers PC, Ponsioen CY, Reitsma JB, et al: Pilot study of probe-based confocal laser endomicroscopy during colonoscopic surveillance of patients with longstanding ulcerative colitis. Endoscopy 43:116-122, 2011

18. Venkatesh K, Cohen M, Evans C, Delaney P, Thomas S, Taylor C, et al: Feasibility of confocal endomicroscopy in the diagnosis of pediatric gastrointestinal disorders. World J Gastroenterol 15:2214-2219, 2009
19. Zehri AH, Ramey W, Georges JF, Mooney MA, Martirosyan NL, Preul MC, et al: Neurosurgical confocal endomicroscopy: A review of contrast agents, confocal systems, and future imaging modalities. Surg Neurol Int 5:60, 2014

\section{Disclosures}

Drs. Eschbacher, Nakaji, Preul, and Spetzler hold US patent No. $9,044,142$ "Surgical optical systems for detecting brain tumors" along with Carl Zeiss Meditec AG. Barrow Neurological Institute has no marketing relationship with Optiscan Pty Ltd or with Carl Zeiss Surgical GmbH for the technology described in this article. Optiscan Pty Ltd and Carl Zeiss Surgical GmbH had no input in the interpretation of imaging or histology. This work was supported in part by a grant and equipment provided by Carl Zeiss Surgical GmbH, Oberkochen, Germany, and by funds from the Newsome Family Endowed Chair in Neurosurgery Research held by Dr. Preul.

\section{Author Contributions}

Conception and design: Preul, Martirosyan, Nakaji. Acquisition of data: Martirosyan, Eschbacher, Kalani, Belykh. Analysis and interpretation of data: Preul, Martirosyan, Eschbacher, Kalani, Turner, Belykh. Drafting the article: Preul, Martirosyan, Turner. Critically revising the article: Preul, Eschbacher, Kalani, Turner, Spetzler, Nakaji. Reviewed submitted version of manuscript: Preul. Statistical analysis: Martirosyan, Kalani, Turner, Belykh. Administrative/technical/material support: Preul, Spetzler, Nakaji. Study supervision: Preul, Spetzler, Nakaji.

\section{Correspondence}

Mark C. Preul, c/o Neuroscience Publications, Barrow Neurological Institute, St. Joseph's Hospital and Medical Center, 350 W. Thomas Rd., Phoenix, AZ 85013.email: neuropub@ dignityhealth.org. 